\title{
CASE REPORT
}

\section{Ureteric sarcoidosis - a rare entity}

\author{
V KALIA, MD, K VISHAL, MD, J S GILL, MBBS and A GILL, MD
}

Delta Heart Center 70-K, Sarabha Nagar Ludhiana, 141001 Punjab, India

\begin{abstract}
Primary ureteric involvement in sarcoidosis is very rare; to our knowledge, only a few cases have been reported in the literature. We present here a rare case of ureteric sarcoidosis presenting with obstructive uropathy.
\end{abstract}

Received 7 July 2009

Revised 10 October 2009

Accepted 13 October 2009

DOI: $10.1259 /$ bjr/17908733

(C) 2010 The British Institute of Radiology
Sarcoidosis is a multisystemic disorder of unknown aetiology characterised by the formation of non-caseating granulomas. This condition can involve any organ system of the body or any group of lymph nodes. The respiratory system is most commonly involved; hilar lymphadenopathy is the most common radiological finding with or without changes of interstitial lung disease. Involvement of the genitourinary system is uncommonly seen, with primary ureteric involvement being rare and presenting a diagnostic challenge.

\section{Case report}

A 43-year-old male presented complaining of left flank pain for the previous 2 months. The pain was not associated with fever, nausea, vomiting or haematuria. General and systemic examination did not reveal any significant abnormality. Laboratory investigations were within normal limits except for mildly elevated blood uric acid levels $\left(7.6 \mathrm{mg} \mathrm{dL}^{-1}\right)$. Urine cytology revealed 10-15 red blood cells and 1-3 pus cells per high-power field. No growth of pyogenic organisms was seen even after $48 \mathrm{~h}$ on urine culture. Ultrasonography of the abdomen showed left hydroureteronephrosis without any evidence of nephrolithiasis. Contrast-enhanced CT of the abdomen showed a mildly enlarged left kidney with marked hydronephrosis (calyceal separation of $26 \mathrm{~mm}$ ). The left renal pelvis was also dilated $(40 \mathrm{~mm}$ in anteroposterior dimension). The left upper ureter was dilated with an irregular soft-tissue density lesion seen in the upper ureter approximately $12 \mathrm{~mm}$ distal to the pelvic ureteric junction. Stranding was seen in the periureteric fat (Figure 1). Multiple discrete lymph nodes with a short-axis diameter of $12 \mathrm{~mm}$ were also noted in the retroperitoneum. After being diagnosed as having left ureteric lesion with hydroureteronephrosis, the patient underwent cystoscopy and flexible ureteroscopy. These investigations did not reveal any additional lesions in the bladder or

Address correspondence to: Dr Vishal Kalia, 464-Sec 8/II, Urban Estate, Karnal, Haryana, India. E-mail: v2k1978@yahoo.co.in ureter. No retrograde pyelography was carried out in this patient. Subsequently, the patient underwent laparoscopic excision of the ureteric lesion with ureteropyelostomy and double J ureteric stents (DJ) stenting. Histopathology of the excised specimen revealed granulomatous inflammation with non-caseating granulomas, a few Schaumann bodies and extensive fibrosis consistent with sarcoidosis. CT of the chest was performed to exclude pulmonary sarcoidosis and did not reveal any parenchymal changes or lymphadenopathy. On follow-up, the DJ stent was removed. There was some residual hydronephrosis on the left side with calyceal separation of 8-9 $\mathrm{mm}$. A follow-up CT scan of the patient's chest revealed a few nodules. Currently, the patient is on steroids and receives regular ultrasound follow-up for hydronephrosis.

\section{Discussion}

Sarcoidosis is a multisystem inflammatory disease characterised by the formation of non-caseating granulomas. Pulmonary manifestations are the initial and most common manifestations seen in approximately $90 \%$ of cases [1]. Sarcoidosis can involve virtually any organ system; however, genitourinary involvement is rarely encountered as an initial manifestation. In the genitourinary system, the kidney is most commonly affected (7$22 \%$ of cases); involvement of the ureter, urethra, testis, epididymis and prostate has rarely been reported [2,3]. Renal manifestations can be in the form of direct renal involvement, like nephrotic syndrome, glomerulonephritis and tubulointerstitial disease, or as a result of abnormal calcium metabolism leading to nephrocalcinosis and renal failure.

We report a rare case of sarcoidosis primarily involving the ureter and presenting as flank pain owing to obstructive uropathy. To our knowledge, only a few case reports of primary ureteric involvement in sarcoidosis have been published in the literature so far [4,5]. In our case, CT showed a soft-tissue density lesion in the upper ureter with periureteric stranding and hydroureteronephrosis. Hydronephrosis in a patient with sarcoidosis 


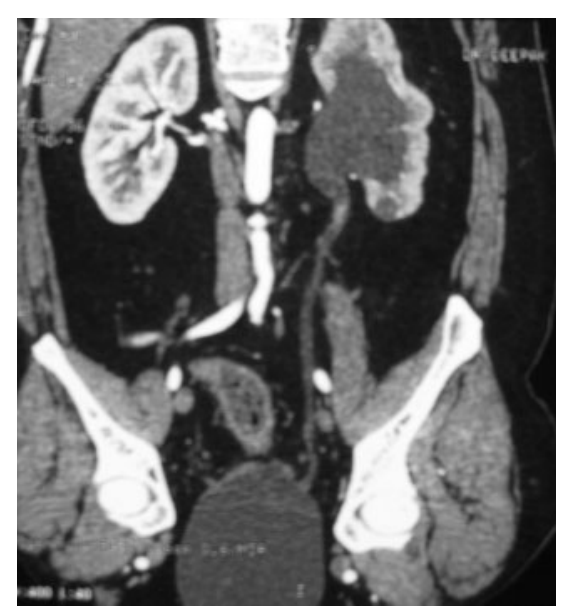

(a)

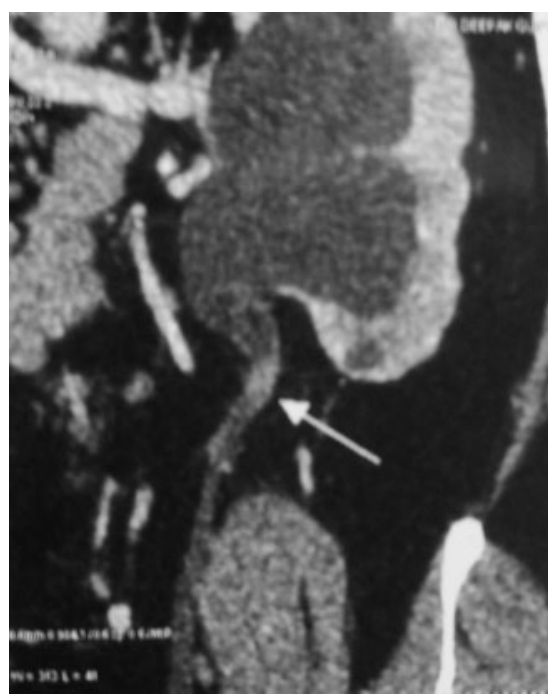

(b)

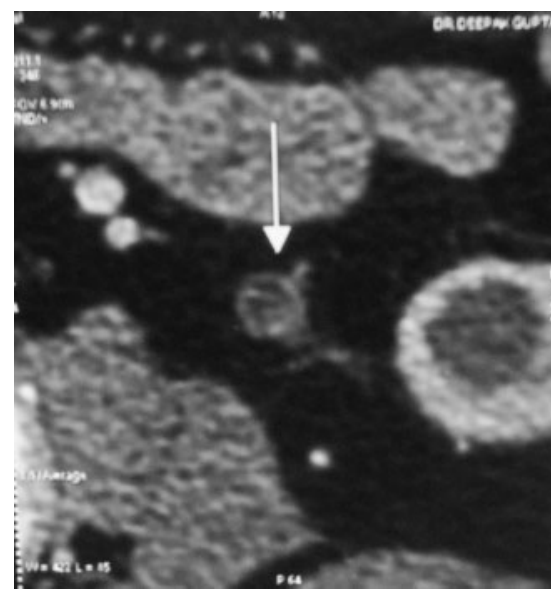

(c)

Figure 1. Contrast-enhanced coronal $(a, b)$ and axial (c) CT images of the abdomen showing a mildly enlarged left kidney with marked hydrouretronephrosis. The left renal pelvis and upper ureter is dilated with an irregular soft-tissue density lesion (arrow) seen in the upper ureter approximately $12 \mathrm{~mm}$ distal to the pelvic ureteric junction. Stranding is seen in the periureteric fat.

can be due to either retroperitoneal lymphadenopathy or primary involvement of the ureter. Although retroperitoneal lymphadenopathy is common in sarcoidosis, hydronephrosis secondary to lymphadenopathy is rare, and primary involvement of the ureter is even rarer $[2,4]$. Imaging plays a pivotal role in diagnosing this entity.

In such cases, CT not only helps in diagnosing the level of obstruction but it can also identify the cause of the obstruction when it is not apparent. Final diagnosis can be made only by pathological examination of the lesion, which shows typical non-caseating granulomas (as seen in our case). However, on imaging, the differential diagnosis of such a lesion includes infections (tuberculosis, histoplasmosis, syphilis, actinomycosis), primary ureteric malignancy, retroperitoneal fibrosis and metastatic disease.

\section{References}

1. Warshauer DM, Lee JK. Imaging manifestations of abdominal sarcoidosis. AJR Am J Roentgenol 2004;182:15-28.

2. Mariano RT, Sussman SK. Sarcoidosis of the ureter. AJR 1998;171:1431.

3. Lynch JP III. Extrapulmonary sarcoid. Semin Respir Infect 1998;13:229-54.

4. Miyazaki E, Tsuda T, Mochizuki A, Sugisaki K, Ando M, Matsumoto T, et al. Sarcoidosis presenting as bilateral hydronephrosis. Intern Med 1996;35:579-82.

5. Perimenis P, Athamasopoulos A, Barbabias G. Sarcoidosis of ureter. Eur Urol 1990;18:307-8. 